\title{
Prevalence of Diffuse Idiopathic Skeletal Hyperostosis in Patients with Spinal Disorders
}

\author{
Hiromitsu Toyoda ${ }^{1}$, Hidetomi Terai $^{1}$, Kentaro Yamada $^{1}$, Akinobu Suzuki $^{1}$, \\ Sho Dohzono ${ }^{2}$, Tomiya Matsumoto ${ }^{3}$, Hiroaki Nakamura ${ }^{1}$ \\ ${ }^{1}$ Department of Orthopaedic Surgery, Osaka City University Graduate School of Medicine, Osaka, Japan \\ ${ }^{2}$ Department Orthopaedic Surgery, Yodogawa Christian Hospital, Osaka, Japan \\ ${ }^{3}$ Department of Orthopaedic Surgery, Osaka Rosai Hospital, Osaka, Japan
}

Study Design: Retrospective cohort study.

Purpose: The purpose of this study was to evaluate the prevalence of diffuse idiopathic skeletal hyperostosis (DISH) in patients with spinal diseases determined by roentgen images of the whole spine.

Overview of Literature: Although several studies have investigated the prevalence of DISH in healthy subjects, no detailed data have been reported on the prevalence of DISH in patients with degenerative spinal disorders.

Methods: Standing whole-spine roentgen images of 345 consecutive patients who underwent surgery in our hospital were obtained. Patients aged $<18$ years or with congenital spinal disease, metastatic spinal tumors, or inflammatory spinal disease were excluded. In total, 281 patients were eligible for inclusion. The presence of DISH was assessed according to Resnick's criteria and Mata's scoring system. The prevalence, location, and numbers of fused vertebral bodies of DISH were recorded.

Results: DISH was present in $25.6 \%$ of patients (72/281). The prevalence of DISH in the 41-49, 50-59, 60-69, 70-79, and $\geq 80$ year age groups was $8.3 \%$ (2/24), 9.8\% (5/51), 16.0\% (12/75), 49.5\% (48/97), and 33.3\% (4/12), respectively; the prevalence increased with age. The average number of fused vertebral bodies was 7.5. More than $80 \%$ of DISH was located from T7 to T11, and more than $95 \%$ of DISH was located at T9/10. Patients with DISH were significantly older (71.1 years vs. 60.9 years, $p<0.05)$, and men were more likely to have DISH than women $(p<0.05)$.

Conclusions: In patients with degenerative spinal diseases with DISH, fused vertebrae were found most frequently in the lower thoracic spine, and their prevalence increased with age. DISH may be an age-related skeletal disorder with a higher overall prevalence in patients with spinal disorders than that in healthy subjects.

Keywords: Spinal diseases; Diffuse idiopathic skeletal hyperostosis; Ankylosing hyperostoses; Forestier's disease

\section{Introduction}

Diffuse idiopathic skeletal hyperostosis (DISH) is a noninflammatory disease, with the principal manifestation being calcification and ossification of the spinal ligaments and regions where tendons and ligaments attach to bone. DISH was first described as senile ankylosing hyperostosis of the spine by Forestier and Rotes-Querol [1] in 1950. During the 1970s, the concept of DISH was extended by Resnick et al. [2] to include extraspinal manifestations.

\footnotetext{
Received Apr 27, 2016; Revised Jun 30, 2016; Accepted Jul 22, 2016

Corresponding author: Hitomitsu Toyoda

Department of Orthopaedic Surgery, Osaka City University Graduate School of Medicine,

1-4-3 Asahi-machi, Abeno-ku, Osaka, Japan

Tel: +81-6-6645-3851, Fax: +81-6-6646-6260, E-mail: h-toyoda@msic.med.osaka-cu.ac.jp
} 
They further advocated three sets of classification criteria for diagnosing this condition. Although several clinical symptoms have been described, including dysphagia, unstable spinal fractures, postsurgical heterotropic ossification, difficult intubation, difficult gastroscopy, aspiration pneumonia, and myelopathy, the most common finding during the physical examination is decreased range of spinal motion, particularly thoracic movement [3-6].

The distinctive finding of DISH is flowing linear calcification and ossification along the anterolateral aspects of the vertebral bodies in the thoracic spine. Complete bridging of the disc space by bone, however, is infrequent in the cervical and lumbar spine [7-13]. Fused spinal segments can develop at the junction of the fused and mobile spine secondary to chronic abnormal stress. This complication manifests radiographically as destruction of intervertebral disc space and nonunion of vertebral fractures, which can lead to neurological disturbances $[4,14]$. Based on the definition of DISH as the radiographic finding of ossification, we hypothesized that the condition might be associated with disc degeneration or hypertrophy of the ligamentum flavum, leading to spinal disorders such as lumbar spinal stenosis. There are few reports of an association between DISH and degenerative spinal disorders that require spinal surgery, whereas there are many published population-based studies that have provided reliable evidence for the prevalence of DISH in the general population. According to previous reports, the prevalence of DISH ranges from $2.9 \%$ to $39.0 \%$ [1,713]. If the prevalence of DISH in a hospital-based cohort of patients who are undergoing spine surgery is higher than that from population-based studies, then the presence of DISH is possible with symptomatic degenerative spinal disorders. The purpose of this study was to evaluate the prevalence and distribution of DISH in Japanese patients with spinal disorders that required surgery and compare the results with those of a previous populationbased study.

\section{Materials and Methods}

A total of 345 consecutive patients who underwent surgical procedures in our hospital were recruited. Patients aged $<18$ years or with congenital spinal disease, metastatic spinal tumors, or inflammatory spinal disease were excluded. All subjects underwent roentgen imaging of the whole spine. A total of 281 patients were eligible for inclusion (Table 1). The study population was divided into

Table 1. Characteristics of the study population ( $n=281$ )

\begin{tabular}{lll}
\hline Level of spinal surgery & & Surgical procedure \\
\hline Cervical spine (87) & CSM (51) & LP (46), PDF (3), ADF (2) \\
\hline & OPLL (11) & LP (11) \\
\hline & CDH (6) & LP (4), ADF (2) \\
& Spinal tumor (5) & Tumor resection (5) \\
\hline CSA (2) & ADF (2) \\
\hline Thoracic spine (28) & Trauma (9) & PDF (9) \\
\hline Non union of VF (15) & VP (13), PDF (2) \\
\hline OPLL (2) & PDF (2) \\
\hline OYL (3) & PD (3) \\
\hline Spinal tumor (5) & Tumor resection (5) \\
\hline Trauma (3) & PDF (2), ADF (1) \\
\hline LSS, DS (122) & PD (80) PDF (42) \\
\hline Spondylosis (6) & PDF (6) \\
\hline LDH (30) & Herniotomy (29), PDF (1) \\
\hline Spinal tumor (7) & Tumor resection (7) \\
\hline
\end{tabular}

CSM, cervical spondylotic myelopathy; LP, laminoplasty; PDF, posterior spinal decompression and fusion surgery; ADF, anterior spinal decomression and fusion surgery; OPLL, ossification of the posterior longitudinal ligament; $\mathrm{CDH}$, Cervical disc herniation; CSA, cervical spondylotic, cervical spondylotic amyotrophy; VF, vertebral fracture; VP, vertebroplasty; OYL, ossification of the yellow ligament; PD, Posterior decompression surgery; LSS, lumbar spinal stenosis; DS, degenerative lumbar spondylolisthesis; LDH, lumbar disc herniation. 
two groups based on the presence or absence of DISH: the DISH group, comprising those individuals who were diagnosed with DISH according to the modified Resnick's criteria and Mata's scoring system, and the non-DISH group.

Each patient signed an informed consent to participate in this cohort study. The study was approved by the Institutional Review Board of Osaka City University (No. 3074).

Standing radiographs of the whole spine were routinely performed in all 281 participants at baseline. All radiographs were reviewed in consensus by two authors (H.T. and K.Y.) with more than 10 years of experience.

The presence of DISH was assessed according to Resnick's criteria and Mata's scoring system. Resnick et al. [2] defined DISH as the presence of four or more vertebral bodies with continuous ossification of the anterior spinal ligaments and the absence of degenerative disc disease. Mata et al. [15] developed a scoring system to grade DISH from 0 to 3 based on the degree of ossification at each disc space level, where $0=$ no ossification, $1=$ ossification without bridging, $2=$ ossification with incomplete bridging, and $3=$ complete bridging of the disc space. In the current study, the presence of DISH was defined according to Mata et al. [15] as a grade of 3 at three or more consecutive disc space levels regardless of extraspinal manifestations. We recorded the prevalence, location, and numbers of fused vertebral bodies of DISH.

Demographic data of the patients included sex, exact age (years), body weight $(\mathrm{kg})$, and height $(\mathrm{cm})$ with reference to the radiographic assessment data. Weight and height were used to calculate the body mass index (BMI) (kilograms per square meter). Information on history of diabetes mellitus and cardiac disease was obtained from self-reports after diagnosis by a physician.

The distribution of baseline characteristics among participants with and without DISH was compared using $\chi^{2}$ tests for categorical variables and $t$ tests for continuous variables (significance set at $p<0.05$ ). Statistical analysis was performed with SPSS ver. 19.0 (SPSS Inc., Chicago, IL, USA).

\section{Results}

\section{Prevalence of DISH}

Seventy-two of 281 patients were diagnosed with DISH, and the overall prevalence of DISH was $25.6 \%$. One hundred sixty-three patients were men (58.0\%) and 118 were women (42.0\%); their mean age was 63.5 years (range, $20-86$ years). The DISH group comprised 49 men (68.1\%) and 23 women (31.9\%); their mean age was 71.1 years (range, 37-84 years). The non-DISH group comprised 114 men (54.5\%) and 95 women (45.5\%); their mean age was 60.9 years (range, $20-86$ years). The prevalence of DISH in the $41-49,50-59,60-69,70-79$, and $\geq 80$ year age groups was $8.3 \%(2 / 24), 9.8 \%$ (5/51), $16 \%$ (12/75), 49.5\% (48/97), and $33.3 \%(4 / 12)$, respectively. The prevalence of DISH increased with age (Fig. 1).

\section{Characteristics of DISH}

Ligamentous ossifications in DISH mainly involved the middle and lower thoracic spine. More than $80 \%$ of ligamentous ossifications in DISH were located from T7 to T11. Ninety-six percent of ligamentous ossifications in DISH included the T9/10 level (Fig. 2). The average number of ligamentous ossifications in DISH was 7.5. The average numbers of ligamentous ossifications in DISH for the $40-49,50-59,60-69,70-79$, and $\geq 80$ year age groups were $4.5 \pm 2.1,6.2 \pm 2.8,6.6 \pm 2.0,7.4 \pm 4.3$, and $6.0 \pm 3.8$, respectively (Fig. 3). The number of ligamentous ossifications in DISH increased with age.

\section{Between-group differences in demographic charac- teristics}

Demographic characteristics and group comparisons between the DISH and non-DISH groups are shown in

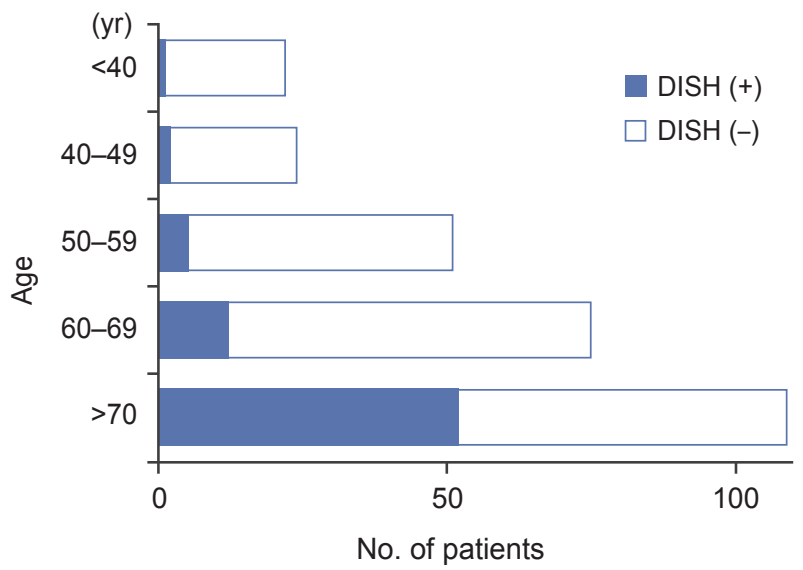

Fig. 1. Bar chart showing the overall percent prevalence of diffuse idiopathic skeletal hyperostosis (DISH) according to age. 


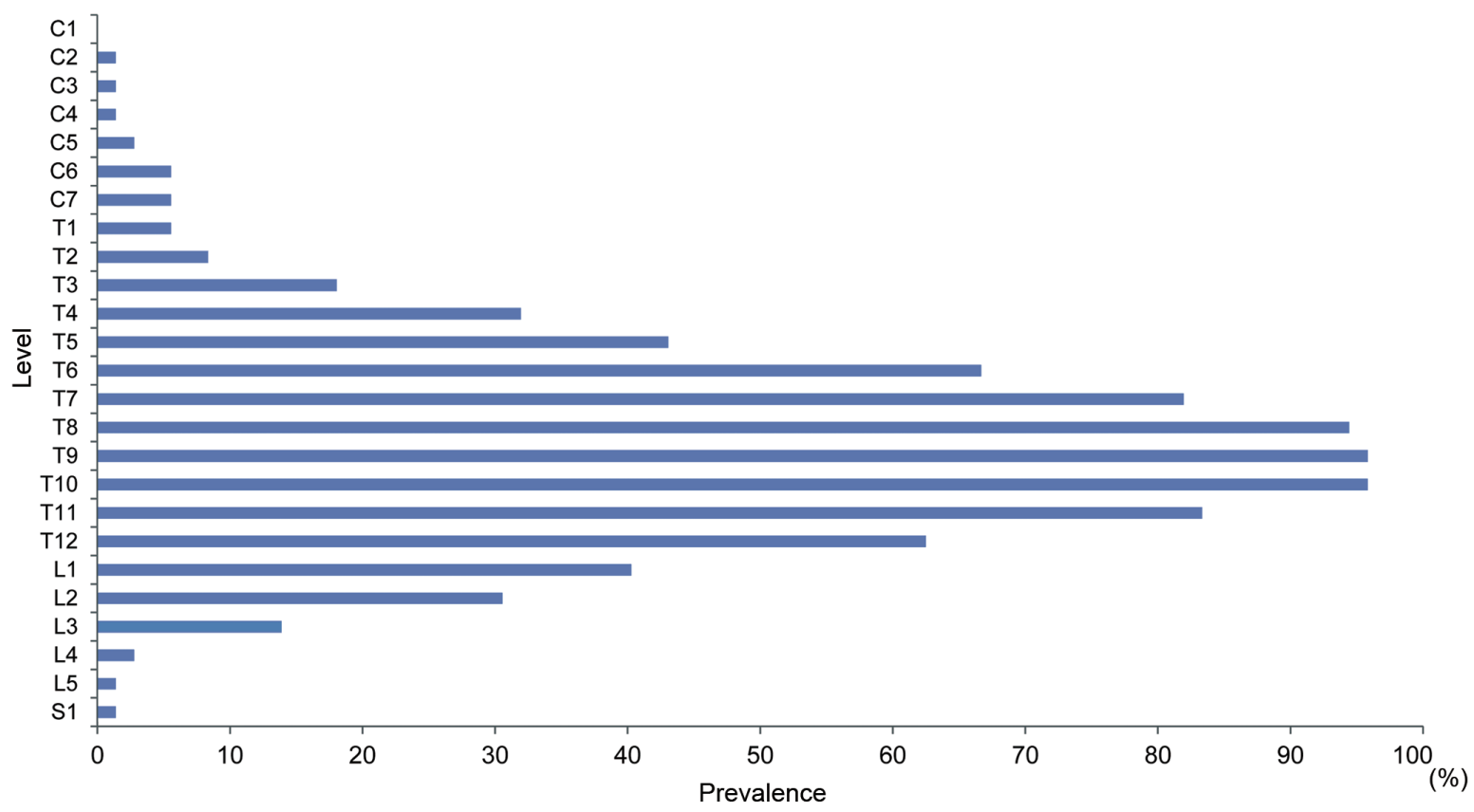

Fig. 2. Manifestations of diffuse idiopathic skeletal hyperostosis per spinal segment in the total study population according to the Mata classification.

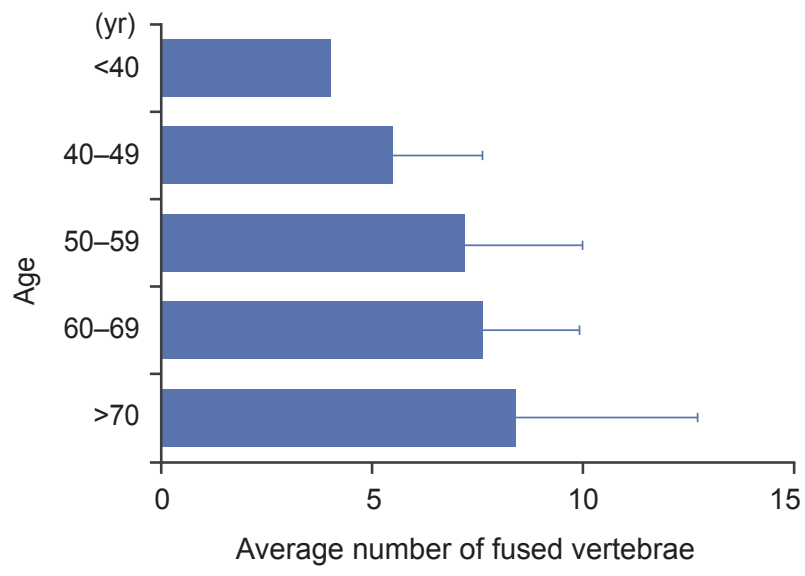

Fig. 3. Bar chart showing the average number of fused vertebrae in the diffuse idiopathic skeletal hyperostosis group according to age.

Table 2. The patients with DISH were significantly older (71.1 years vs. 60.9 years, $p<0.05$ ), and men were more likely to have DISH than women. Body weight and BMI were significantly higher in the DISH group than in the non-DISH group (mean body weight, $62.4 \mathrm{~kg}$ vs. $57.9 \mathrm{~kg}$; mean BMI, $24.5 \mathrm{~kg} / \mathrm{m}^{2}$ vs. $22.9 \mathrm{~kg} / \mathrm{m}^{2} ; p<0.05$ for both comparisons). Although there were no significant correlations between the presence of DISH and the other parameters, such as diabetes mellitus, cardiac disease, level of surgery, and primary spinal disorders, lumbar spinal stenosis was more prevalent in patients with DISH who were under 65 years of age.

\section{Discussion}

We reviewed 281 patients with various types of spinal disorders. The overall prevalence of DISH according to Resnick's criteria was $26 \%$, and the prevalence increased to $48 \%$ in patients who were over 70 years of age. This is the first report in which the prevalence of DISH in patients with spinal disorders was studied using roentgen images of the whole spine. The reported prevalence of DISH ranges from $2.9 \%$ in Korea to $38 \%$ in the United States, and the overall prevalence of DISH was reportedly higher in a predominantly white population over the age of 50 years, with lower prevalence rates in black, native American, and Asian populations [1,7-13,16-18]. Because the clinical manifestations of DISH are minor, most previous cohort studies used chest radiographs to screen subjects who visited the hospital, regardless of their illness. The prevalence depends substantially on the classification criteria and screening methods used. Although the pathogenesis of DISH is not fully understood, several authors have proposed that the reported prevalence may well increase in the coming decades because of the relationship between DISH and modern lifestyle-related diseases, such as obesity and metabolic syndrome $[12,19]$. 
Table 2. Differences between the DISH group and non-DISH group ( $\mathrm{n}=281$ )

\begin{tabular}{|c|c|c|c|}
\hline Baseline characteristic & DISH group (n=72) & Non-DISH group (n=209) & $p$-value \\
\hline Age (yr) & $71.1(8.6)$ & $60.9(14.6)$ & $<0.001$ \\
\hline Height (cm) & $159.6(9.4)$ & $158.8(10.0)$ & 0.586 \\
\hline Weight (kg) & $62.4(13.3)$ & $57.9(11.9)$ & 0.009 \\
\hline Body mass index & $24.5(3.8)$ & $22.8(3.5)$ & 0.001 \\
\hline Sex, men & $49(68.1)$ & $114(54.5)$ & 0.045 \\
\hline \multicolumn{4}{|l|}{ Diabetes mellitus } \\
\hline Age $<65$ yr & $1 / 10(10.0)$ & $12 / 105(11.4)$ & 0.891 \\
\hline Age $>65$ yr & $15 / 62(24.2)$ & 18/104 (17.3) & 0.282 \\
\hline \multicolumn{4}{|l|}{ Cardiac disease } \\
\hline Age $<65$ yr & $3 / 10(30.0)$ & $16 / 105(15.2)$ & 0.230 \\
\hline Age $>65 \mathrm{yr}$ & $32 / 62(51.6)$ & 41/104 (39.4) & 0.126 \\
\hline Level of spinal surgery & & & 0.162 \\
\hline Cervical spine & 16 & 71 & \\
\hline Thoracic spine & 9 & 19 & \\
\hline Lumbar spine & 47 & 119 & \\
\hline \multicolumn{4}{|l|}{ OPLL or OYL } \\
\hline Age $<65$ yr & $2 / 10(20.0)$ & $7 / 105(6.7)$ & 0.133 \\
\hline Age $>65$ yr & $3 / 62(4.8)$ & $3 / 104(2.9)$ & 0.514 \\
\hline \multicolumn{4}{|l|}{ Non union of VF } \\
\hline Age $<65$ yr & $0 / 10(0)$ & $1 / 104(0.9)$ & 0.097 \\
\hline Age >65 yr & $6 / 62(9.7)$ & $7 / 105(6.7)$ & 0.555 \\
\hline \multicolumn{4}{|l|}{ LSS } \\
\hline Age $<65$ yr & $5 / 10(50.0)$ & 28/104 (26.9) & 0.124 \\
\hline Age $>65 y r$ & 37/62 (59.7) & 56/105 (53.3) & 0.519 \\
\hline
\end{tabular}

Values are presented as mean \pm standard deviation or number (\%).

DISH, diffuse idiopathic skeletal hyperostosis; OPLL, ossification of the posterior longitudinal ligament; OYL, ossification of the yellow ligament; VF, Vertebral fracture; LSS, Lumbar spinal stenosis.

Three major articles have been published regarding the prevalence of DISH in a Japanese study. Kagotani et al. [10] conducted a survey, known as the Research on Osteoarthritis/Osteoporosis Against Disability (ROAD) study, using a population-based cohort in Japan. They reported that radiographically the prevalence of DISH was $10.8 \%$ (22.0\% in men and $4.8 \%$ in women). They also reported that the prevalence of DISH was significantly higher in older participants and was significantly associated with lumbar spondylosis and severe knee osteoarthritis [10]. Fujimori et al. [9] reported that the prevalence of DISH in self-paying participants in a preventive cancer screening by computed tomography (CT) was $12 \%$ (16.0\% in men and $6.2 \%$ in women). Hirasawa et al. [8] investigated the prevalence of DISH by both reconstructed CT and plain radiography of the breast. They found that the prevalence of DISH based on CT was $27.1 \%$, which was higher than that found by radiography. Compared with these previous reports, the higher prevalence of DISH reported here was likely due to the subjects' older age and the fact that we investigated only patients with spinal disorders. We considered that it was important to investigate the prevalence of DISH in a selected population that requires spinal surgery, because the specific biomechanical condition of DISH could influence the selection of treatment.

Most clinical symptoms of DISH are mild, although complaints such as dysphagia and airway obstruction at the cervical level and serious complications, including 
myelopathy and unstable thoracolumbar fractures after trauma, are being reported with increasing frequency [3$5,20]$. Not only acute injury but also chronic, continuous stress at the junction of fused and nonfused sites of the spine in DISH can cause spinal disorders such as nonunion of the vertebral body and lumbar spinal stenosis (LSS). Quagliano et al. [14] suggested that there were two types of symptomatic intervertebral pseudarthrosis with DISH: that which occurred after acute fracture and that which was the result of cumulative stress at the junction of mobile and immobile vertebral segments. Miyamoto et al. [21] also noted that patients with an intervertebral space associated with DISH presented with severe back pain in the absence of a history of trauma. Although a crosssectional study cannot evaluate cause and effect, the magnified load relative to that of the nonfused lower lumbar segments might affect the onset, or increase the severity, of degeneration and LSS.

The management of spinal disorders with DISH remains a formidable challenge that requires a thorough understanding of DISH-specific biomechanics of the spine and the mechanism of injury as it relates to the concept of spinal stability. There are several treatment precautions for spinal disorders with DISH. The first precaution is to avoid increased stress or hypermobility at the adjacent segment of the fused spine. Numerous biomechanical studies have confirmed that the fusion process imposes significant amounts of stress at the adjacent segment [2224]. Important factors leading to adjacent-segment motion, stress, and degenerative changes after fusion surgery include stiffness, sagittal alignment, and the number of fused segments. Considering these factors, we chose a relatively long fusion area to enable us to obtain sufficient stability against the large forces undoubtedly being applied to the junction of the mobile and immobile spine segments. Caron et al. [6] recommended posterior segmental fixation extending a minimum of three levels above and below the injury for vertebral fractures in patients with DISH. Although there are few reports of surgical strategies for LSS with DISH, Chi et al. [25] reported that posterior lumbar interbody fusion may be an option for surgical treatment of symptomatic nonunited lumbar segments at the caudal end of a fused spine in patients with DISH who are unresponsive to conservative treatment. Otsuki et al. [26] investigated patients with surgery-free survival or the need for further surgery for pseudarthrosis and/or adjacent segment disease after short-segment lum- bar interbody fusion. They reported that DISH and twolevel fusion were significant independent predictors of the need for further surgery, even if ossification is seen only in the thoracic to upper lumbar region [26]. Long fusion may be one of the surgical strategies for spinal disorders with DISH; however, the problem would remain that long fusion might decrease the mobile spinal segment of DISH.

The second precaution is that the fused spine is more prone to fracture than a normal spine [27]. These fused segments, which are pathognomonic for AS and DISH, act as long lever arms that increase the forces applied to the spine, which, in many instances, may already be osteoporotic secondary to abnormal bone production [28]. Paravertebral calcifications of DISH may lead to overestimation of bone mineral density (BMD) values by dualenergy X-ray absorptiometry. Several studies, however, have documented an increased risk of vertebral fractures in people with DISH $[4,6,27,29,30]$. Diederichs et al. [27] found that decreased BMD in patients with DISH could contribute to an increased risk of vertebral fractures. We should consider that the holding power of screws in the vertebrae of patients with DISH may decrease with decreasing BMD.

The third precaution is that the population of patients with DISH includes individuals of advanced age. We should consider that DISH is also associated with vertebral osteoporosis and the risk of complications associated with the extensive surgery. Considering the potential increase in the prevalence of DISH, we should pay attention to this condition when we create a treatment plan for patients with spinal disorders.

This study has several limitations. First, because it was a cross-sectional study of patients with spinal disorders who required spinal surgery and we did not have data on healthy subjects, we compared our data with those from previous reports. Although a cross-sectional study cannot evaluate cause and effect, our results show that the presence of DISH is possible with the onset of LSS. Second, the sample size of this study was not sufficient for generalizability. Further studies based on a larger sample should be performed to confirm the present results. Third, the prevalence of DISH depends substantially on the classification criteria and screening methods used. It is possible that a radiography-based study might find a lower prevalence than would a CT-based study [8]. Further studies based on whole-spine CT should be performed to confirm the present results. A radiography-based study, however, 
might be more useful than a CT-based study because of the lower radiation dose and greater convenience of radiography.

\section{Conclusions}

DISH begins at the lower level of the thoracic spine, and its prevalence increases with age. DISH may be an agerelated skeletal disorder with an overall prevalence higher in patients with spinal disorders than in healthy subjects.

\section{Conflict of Interest}

No potential conflict of interest relevant to this article was reported.

\section{ORCID}

Hiromitsu Toyoda: 0000-0002-3126-0046

Kentaro Yamada: 0000-0001-5454-4554

Hiroaki Nakamura: 0000-0002-0593-8810

\section{References}

1. Forestier J, Rotes-Querol J. Senile ankylosing hyperostosis of the spine. Ann Rheum Dis 1950;9:321-30.

2. Resnick D, Shaul SR, Robins JM. Diffuse idiopathic skeletal hyperostosis (DISH): Forestier's disease with extraspinal manifestations. Radiology 1975;115:51324.

3. Fox TP, Desai MK, Cavenagh T, Mew E. Diffuse idiopathic skeletal hyperostosis: a rare cause of dysphagia and dysphonia. BMJ Case Rep 2013;2013: bcr2013008978.

4. Westerveld LA, Verlaan JJ, Oner FC. Spinal fractures in patients with ankylosing spinal disorders: a systematic review of the literature on treatment, neurological status and complications. Eur Spine J 2009; 18:145-56.

5. Nascimento FA, Gatto LA, Lages RO, Neto HM, Demartini Z, Koppe GL. Diffuse idiopathic skeletal hyperostosis: a review. Surg Neurol Int 2014;5(Suppl 3):S122-5.

6. Caron T, Bransford R, Nguyen Q, Agel J, Chapman J, Bellabarba C. Spine fractures in patients with ankylosing spinal disorders. Spine (Phila Pa 1976) 2010; 35:E458-64.
7. Kiss C, O’Neill TW, Mituszova M, Szilagyi M, Poor G. The prevalence of diffuse idiopathic skeletal hyperostosis in a population-based study in Hungary. Scand J Rheumatol 2002;31:226-9.

8. Hirasawa A, Wakao N, Kamiya M, et al. The prevalence of diffuse idiopathic skeletal hyperostosis in Japan: the first report of measurement by CT and review of the literature. J Orthop Sci 2016;21:287-90.

9. Fujimori T, Watabe T, Iwamoto Y, Hamada S, Iwasaki M, Oda T. Prevalence, concomitance, and distribution of ossification of the spinal ligaments: results of whole spine CT scans in 1500 Japanese patients. Spine (Phila Pa 1976) 2016;41:1668-76.

10. Kagotani R, Yoshida M, Muraki S, et al. Prevalence of diffuse idiopathic skeletal hyperostosis (DISH) of the whole spine and its association with lumbar spondylosis and knee osteoarthritis: the ROAD study. J Bone Miner Metab 2015;33:221-9.

11. Kim SK, Choi BR, Kim CG, et al. The prevalence of diffuse idiopathic skeletal hyperostosis in Korea. J Rheumatol 2004;31:2032-5.

12. Weinfeld RM, Olson PN, Maki DD, Griffiths HJ. The prevalence of diffuse idiopathic skeletal hyperostosis (DISH) in two large American Midwest metropolitan hospital populations. Skeletal Radiol 1997;26:222-5.

13. Cassim B, Mody GM, Rubin DL. The prevalence of diffuse idiopathic skeletal hyperostosis in African blacks. Br J Rheumatol 1990;29:131-2.

14. Quagliano PV, Hayes CW, Palmer WE. Vertebral pseudoarthrosis associated with diffuse idiopathic skeletal hyperostosis. Skeletal Radiol 1994;23:353-5.

15. Mata S, Chhem RK, Fortin PR, Joseph L, Esdaile JM. Comprehensive radiographic evaluation of diffuse idiopathic skeletal hyperostosis: development and interrater reliability of a scoring system. Semin Arthritis Rheum 1998;28:88-96.

16. Julkunen H, Heinonen OP, Pyorala K. Hyperostosis of the spine in an adult population: its relation to hyperglycaemia and obesity. Ann Rheum Dis 1971;30: 605-12.

17. Bloom RA. The prevalence of ankylosing hyperostosis in a Jerusalem population: with description of a method of grading the extent of the disease. Scand J Rheumatol 1984;13:181-9.

18. Westerveld LA, van Ufford HM, Verlaan JJ, Oner FC. The prevalence of diffuse idiopathic skeletal hyperostosis in an outpatient population in The Netherlands. 
J Rheumatol 2008;35:1635-8.

19. Kiss C, Szilagyi M, Paksy A, Poor G. Risk factors for diffuse idiopathic skeletal hyperostosis: a case-control study. Rheumatology (Oxford) 2002;41:27-30.

20. Mader R. Clinical manifestations of diffuse idiopathic skeletal hyperostosis of the cervical spine. Semin Arthritis Rheum 2002;32:130-5.

21. Miyamoto K, Shimizu K, Arimoto R, et al. Spontaneous symptomatic pseudoarthrosis at the T11-T12 intervertebral space with diffuse idiopathic skeletal hyperostosis: a case report. Spine (Phila Pa 1976) 2003; 28:E320-2.

22. Ames CP, Acosta FL Jr, Chi J, et al. Biomechanical comparison of posterior lumbar interbody fusion and transforaminal lumbar interbody fusion performed at 1 and 2 levels. Spine (Phila Pa 1976) 2005;30:E5626.

23. Kumar MN, Baklanov A, Chopin D. Correlation between sagittal plane changes and adjacent segment degeneration following lumbar spine fusion. Eur Spine J 2001;10:314-9.

24. Sudo H, Oda I, Abumi K, Ito M, Kotani Y, Minami A. Biomechanical study on the effect of five different lumbar reconstruction techniques on adjacent-level intradiscal pressure and lamina strain. J Neurosurg Spine 2006;5:150-5.

25. Chi D, Miyamoto K, Hosoe H, et al. Symptomatic lumbar mobile segment with spinal canal stenosis in a fused spine associated with diffused idiopathic skeletal hyperostosis. Spine J 2008;8:1019-23.

26. Otsuki B, Fujibayashi S, Takemoto M, Kimura H, Shimizu T, Matsuda S. Diffuse idiopathic skeletal hyperostosis (DISH) is a risk factor for further surgery in short-segment lumbar interbody fusion. Eur Spine J 2015;24:2514-9.

27. Diederichs G, Engelken F, Marshall LM, et al. Diffuse idiopathic skeletal hyperostosis (DISH): relation to vertebral fractures and bone density. Osteoporos Int 2011;22:1789-97.

28. Vosse D, Landewe R, van der Heijde D, van der Linden S, van Staa TP, Geusens P. Ankylosing spondylitis and the risk of fracture: results from a large primary care-based nested case-control study. Ann Rheum Dis 2009;68:1839-42.

29. Schreiber JJ, Anderson PA, Rosas HG, Buchholz AL, $\mathrm{Au}$ AG. Hounsfield units for assessing bone mineral density and strength: a tool for osteoporosis management. J Bone Joint Surg Am 2011;93:1057-63.

30. Westerveld LA, van Bemmel JC, Dhert WJ, Oner FC, Verlaan JJ. Clinical outcome after traumatic spinal fractures in patients with ankylosing spinal disorders compared with control patients. Spine J 2014;14:72940. 